\title{
Efeitos da fotobiomodulação no tratamento de úlceras por pressão: Revisão
}

\section{integrativa}

\author{
Effects of photobiomodulation in the treatment of pressure ulcers: Integrative review \\ Efectos de la fotobiomodulación en el tratamiento de las úlceras por presión: Revisión integradora
}

Recebido: 02/02/2021 | Revisado: 10/02/2021 | Aceito: 11/02/2021 | Publicado: 17/02/2021

Suellen Pereira Rodrigues Macedo
ORCID: https://orcid.org/0000-0002-4147-1234
Universidade Brasil, Brasil
E-mail: suenferm @ hotmail.com
Mara Soares de Almeida Mota
Universidade Brasil, Brasil
ORCID: https://orcid.org/0000-0002-2068-3481
E-mail: mara.almeida06@ gmail.com
Caroline Ferreira Fagundes
ORCID: https://orcid.org/0000-0002-9928-2065
Universidade do Estado de Mato Grosso, Brasil
E-mail: caroline.fagundes@ unemat.br
Matheus Rodrigues de Souza
ORCID: https://orcid.org/0000-0001-5517-0777
Universidade do Estado de Mato Grosso, Brasil
E-mail: matheus.rodrigues@ unemat.br
Ricardo Scarparo Navarro
ORCID: https://orcid.org/0000-0003-4351-8048
Universidade Brasil, Brasil
E-mail: ricardosnavarro@ gmail.com

\begin{abstract}
Resumo
Úlceras por pressão (UP) são importantes causas de morbidade, além de possuir alto custo ao sistema de saúde, agrega um risco adicional para os portadores pois impõe a perda da barreira constitucional da pele. A fotobiomodulação com laser de baixa potência (FBM) mostra efetivas ações analgésica, antinflamatória e reparação dos tecidos. O objetivo do presente estudo foi avaliar a eficácia da FBM no tratamento de UP a partir de uma revisão integrativa norteada pela pergunta "Qual a eficácia da fotobiomodulação no tratamento de úlceras por pressão?". O estudo incluiu sete artigos selecionados nas bases de dados Scielo, PubMed e LILACS entre o período de 2015 e 2020. Após a leitura, análise e interpretação dos dados, pôde-se verificar que, apesar de haver poucos estudos recentes sobre a terapia a laser na temática da lesão por pressão, os artigos elucidam que a terapia com laser de baixa potência é uma modalidade promissora de tratamento, que proporciona redução do tempo de cicatrização e melhora no aspecto das feridas. Contudo, apesar de diversos estudos demonstrarem os efeitos benéficos desta, ainda não há consenso e protocolos específicos definidos que determinem parâmetros de irradiação, como comprimento de onda, energia, fluência, potência, irradiância, duração do pulso e intervalos entre as sessões de tratamento.
\end{abstract}

Palavras-chave: Úlcera por pressão; Laser de baixa potência; Fotobiomodulação; Cicatrização de feridas.

\begin{abstract}
Pressure ulcers (PU) are important causes of morbidity, in addition to having a high cost to the health system, it adds an additional risk to patients because it imposes the loss of the constitutional skin barrier. Photobiomodulation with low power laser (FBM) shows effective analgesic, anti-inflammatory and tissue repair actions. The aim of the present study was to evaluate the effectiveness of FBM in the treatment of PU based on an integrative review guided by the question "How effective is photobiomodulation in the treatment of pressure ulcers?". The study included seven articles selected from the Scielo, PubMed and LILACS databases between 2015 and 2020. After reading, analyzing and interpreting the data, it was possible to verify that, although there are few recent studies on laser therapy on the subject of pressure injury, the articles elucidate that low-level laser therapy is a promising treatment modality, which reduces the healing time and improves the appearance of wounds. However, although several studies have demonstrated its beneficial effects, there is still no consensus and specific protocols defined that determine irradiation parameters, such as wavelength, energy, fluency, power, irradiance, pulse duration and intervals between treatment sessions.
\end{abstract}

Keywords: Pressure ulcer; Low level laser therapy; Photobiomodulation; Wound healing. 


\begin{abstract}
Resumen
Las úlceras por presión (UPP) son importantes causas de morbilidad, además de tener un alto costo para el sistema de salud, agrega un riesgo adicional para los pacientes porque impone la pérdida de la barrera cutánea constitucional. La fotobiomodulación con láser de baja potencia (FBM) muestra acciones analgésicas, antiinflamatorias y reparadoras de tejidos eficaces. El objetivo del presente estudio fue evaluar la efectividad de la FBM en el tratamiento de la UP a partir de una revisión integradora guiada por la pregunta "¿Qué tan efectiva es la fotobiomodulación en el tratamiento de las úlceras por presión?". El estudio incluyó siete artículos seleccionados de las bases de datos Scielo, PubMed y LILACS entre 2015 y 2020. Después de leer, analizar e interpretar los datos, se pudo constatar que, aunque existen pocos estudios recientes sobre la terapia con láser en el tema de lesión por presión, los artículos aclaran que la terapia con láser de baja intensidad es una modalidad de tratamiento prometedora, que reduce el tiempo de cicatrización y mejora la apariencia de las heridas. Sin embargo, aunque varios estudios han demostrado sus efectos beneficiosos, aún no existe consenso y se definen protocolos específicos que determinen parámetros de irradiación, como longitud de onda, energía, fluidez, potencia, irradiancia, duración del pulso e intervalos entre sesiones de tratamiento.
\end{abstract}

Palabras clave: Úlcera de presión; Terapia con láser de bajo nível; Fotobiomodulación; Cicatrización de la herida.

\title{
1. Introduçãa
}

Úlcera por pressão (UP) é definida como uma área de pele e/ou tecido lesionado profundamente na pele, geralmente sobre uma proeminência óssea, resultante de pressão prolongada ou intensa, isolada ou combinada com forças de cisalhamento (García-Fernández et al., 2014). UP é uma patologia complexa e multifatorial, desencadeada por fatores extrínsecos como a presença do indivíduo na mesma posição por períodos prolongados, e fatores intrínsecos como desnutrição, insuficiência vasomotora, vasoconstrição periférica, alterações no índice de massa corporal, presença de doenças crônicas, incontinência urinária e fecal (Bhattacharya \& Mishra, 2015; García-Fernández et al., 2014). A idade é um dos fatores intrínsecos que contribui para o desencadeamento da úlcera por pressão, sendo que pacientes de 60 a 84 anos possuem 2,3 vezes mais chance de desenvolver UP (Strazzieri-Pulido et al., 2019). As taxas de prevalência podem variar de $16,9 \%$ a $23,8 \%$ e taxas de incidência de 3,4\% a 25,9\% em diferentes ambientes de cuidados (Mustoe et al., 2006; Sardo et al., 2016; Strazzieri-Pulido et al., 2019).

Em úlceras crônicas, a principal causa da persistência da ferida é colonização e proliferação bacteriana (Brauncajs et al., 2018; Cannon \& Cannon, 2004). A infecção bacteriana ocorre na forma de biofilmes em feridas, que são agregados de bactérias incorporadas em uma matriz de substância polimérica extracelular auto secretada (Brauncajs et al., 2018; Thomé Lima et al., 2019). As bactérias em biofilmes se comportam de maneira diferente quando comparada à forma livre, dificultando o manejo terapêutico das úlceras (Qaseem et al., 2015).

Algumas UP são evitáveis, entretanto, fatores como imobilidade física, que torna o indivíduo mais suscetível, presença de déficit neurológico e redução da percepção sensorial, são fatores que dificultam a implementação de medidas preventivas (Bhattacharya \& Mishra, 2015; National Pressure Ulcer Advisory and Pan Pacific Pressure Injury Alliance, 2015). Feridas crônicas, como úlceras de pressão, apresentam uma interrupção do processo de cicatrização, na cronificação dessas feridas as fases do processo de cicatrização (inflamatória, proliferativa e remodelação) se interpõem (Bauer et al., 2016; Wada et al., 2010).

Nesse caso, a fase inflamatória é estagnada pela isquemia devido à pressão prolongada, gerando insuficiência vascular nos tecidos subjacentes, com diminuição da oxigenação e consequente redução das células responsáveis pela resposta inflamatória que medeiam o processo de cicatrização (Taradaj et al., 2018). Na fase proliferativa, os fibroblastos perdem a capacidade de divisão e produção de colágeno e ocorre a diminuição dos fatores de crescimento tecidual; na fase de remodelação, a síntese de colágeno diminui com consequente redução da força de tração, o que torna o tecido mais propenso à ruptura após sua restauração (Zhao et al., 2016).

Apesar de sua importância, atualmente, não existe tratamento padrão-ouro preconizado para UP (Lima \& Guerra, 2011), apesar disso, temos consensos e guidelines com instruções e recomendações para prevenção, diagnóstico e tratamento 
(Advisory, 1989; Qaseem et al., 2015). O curativo com diferentes coberturas é o tratamento mais empreendido, porém o benefício no processo de cicatrização a médio e longo prazo depende da extensão e profundidade das úlceras e do estado clínico do paciente. Neste sentido, com intuito de acelerar o processo de cicatrização das lesões, novos tratamentos são analisados (Mervis \& Phillips, 2019; Mosca et al., 2019).

Dentre as terapias não farmacológicas, cabe ressaltar a Fotobiomodulação (FBM) promovida pela terapia com Laser de baixa potência (LBP), descrita na literatura como uma opção de tratamento promissora para feridas abertas (Lucas et al., 2003; Palagi et al., 2015; Qaseem et al., 2015).

A Fotobiomodulação (FBM) tem sido aplicada no tratamento de úlceras e feridas crônicas com significativos resultados positivos, cujos efeitos fotoquímicos, fotofísicos e fotobiológicos capazes de alterar o comportamento celular consequentemente, favorecendo a reparação tecidual (Castro et al., 2020; Health, 2019). Diante disso, a ação do laser favorece a oxigenação celular da região lesada através da neoangiogênese pelo aumento da quantidade de capilares, acelerando o reparo dos tecidos lesados (Hernández et al., 2015).

Assim, levando-se em consideração estudos recentes que sugerem que a FBM é uma terapia que pode complementar o manejo terapêutico das UP, com benefício potencial de acelerar o processo de cicatrização (Taly et al., 2004), aumentar o tecido de granulação (Li et al., 2018), reduzir a ferida (Thomé Lima et al., 2019), porém sem padronização ou sistematização do uso (Castro et al., 2020; Monteiro et al., 2020).

Tendo isso em vista, o objetivo do presente estudo é realizar uma revisão integrativa para avaliar os efeitos da fotobiomodulação com laser de baixa potência no tratamento das UP.

\section{Metodologia}

Trata-se de uma revisão integrativa da literatura, explorando dados qualitativos de estudos por meio de uma busca sistemática a respeito do uso de laser de baixa intensidade no tratamento de úlceras por pressão. O presente estudo foi desenvolvido seguindo seis etapas, conforme norteado por alguns autores (Botelho et al., 2011; De Souza et al., 2019; Pereira et al., 2018).

\section{Identificação do tema e formulação da pergunta norteadora}

A questão de pesquisa foi formulada a partir da utilização da estratégia PICOS (Higgins \& Green., 2011), que representa um acrônimo do inglês Population (paciente ou problema), Intervention (Intervenção), Comparison (Comparação), Outcomes (Desfecho) e Study design (Desenho do estudo), a partir da aplicação da estratégia, definiu-se como pergunta norteadora: "Qual a eficácia da fotobiomodulação no tratamento de úlceras por pressão?".

A estratégia de busca incluiu os seguintes descritores: (pressure ulcer; pressure ulcers; ulcer pressure; ulcers pressure; bed sore; bed sores; bedsore; bedsores; pressure sore; pressure sores; decubitus ulcer; decubius ulcers) AND (photobiomodulation; low-level laser therapy; LLLT; low-level light therapy; low-power laser therapy; laser irradiation; laser treatment; phototherapy; laser phototherapy) com seus respectivos termos correspondents em Português e Espanhol. O operador booleano OR foi aplicado entre os termos. A busca foi realizada nas bases de dados PubMed, Medical Literature Analysis and Retrieval System Online (MEDLINE), Literatura Latino-Americana e do Caribe em Ciências da Saúde (LILACS) e Scientific Electronic Library Online (Scielo).

\section{Estabelecimento de critérios de inclusão e exclusão}

Foram estabelecidos como critérios de inclusão: estudos publicados no corte temporal entre janeiro de 2015 a 2020 em Inglês, Português ou Espanhol, analisando ou relatando artigos em pacientes adultos e idosos com úlcera por pressão, 
independente do grau, em qualquer ambiente de cuidado (hospitalar, clínico, ambulatorial ou domiciliar). Em que um dos grupos de pacientes, obrigatoriamente, fossem tratados com laser de baixa intensidade, sendo considerada a variedade de parâmetros de irradiação, tal como o comprimento de onda, energia potência, tempo e intervalo entre as irradiações. Foram excluídos aqueles estudos duplicados, in vitro e pesquisa em animais, e que fossem publicados fora do período analisado.

\section{Identificação dos estudos pré-selecionados e selecionados}

A seleção dos estudos foi realizada por dois revisores cegos e independentes (S. P. R. M. e C. F. F.), em caso de dúvida ou discordância um terceiro autor (R. S. N.) deu a decisão final. Na primeira etapa, foram lidos os títulos e resumos dos estudos encontrados a partir da busca descrita no item anterior, sendo aplicado os critérios de elegibilidade previamente definidos nesta revisão. Os estudos selecionados na primeira etapa, foram lidos integralmente, sendo novamente aplicado os critérios de elegibilidade, determinando, então, a inclusão ou não na pesquisa.

\section{Categorização dos estudos selecionados}

Após avaliar e obter consenso sobre os estudos incluídos, os dois pesquisadores cegos extraíram os dados de forma independente para um formulário de extração de dados padronizados. A base de dados final utilizada para a análise contém: Título do estudo, ano da publicação, autores, delineamento da pesquisa, revista, objetivo, método e conclusões.

Esses dados foram sistematizados, individualmente, em uma planilha de dados, da qual foi extraída parte dos dados que foram organizados e apresentados em duas categorias de descrição e análise, respondendo aos objetivos do estudo. A primeira categoria, mais descritiva, compreende características gerais das publicações, descrevendo os textos selecionados, no que se refere a título, autoria e data de publicação. A segunda categoria discorre sobre o delineamento da pesquisa, revista de publicação, objetivos do estudo e método.

\section{Análise e interpretação dos resultados}

Nesta etapa, a partir da interpretação e síntese dos resultados, comparam-se os dados evidenciados na análise dos artigos ao referencial teórico. Além de identificar possíveis lacunas dos referenciais teóricos, é possível delimitar características dos estudos com intuito de compreender a eficácia do tratamento proposto, e compreender as características das terapias utilizadas, avaliando a eficácia descrita das mesmas.

\section{Sintese da revisão dos dados}

A apresentação da síntese da revisão dos dados é demonstrada a seguir, objetivando responder à pergunta norteadora.

\section{Resultados e Discussão}

Após a seleção e leitura dos artigos resultantes da busca nas bases de dados através dos descritores associados, realizou-se uma seleção detalhada na Figura 1. Cabe destacar que os artigos foram selecionados e analisados visando responder a pergunta norteadora do estudo. Um total de sete artigos foram identificados e selecionados a partir de uma revisão extensa seguindo os passos metodológicos supracitados (De Oliveira Bernardes \& Regina Jurado, 2018; Hernández et al., 2015; Machado et al., 2017; Palagi et al., 2015; Polachini et al., 2019; Taradaj et al., 2018). 
Figura 1. Fluxograma de seleção dos estudos.
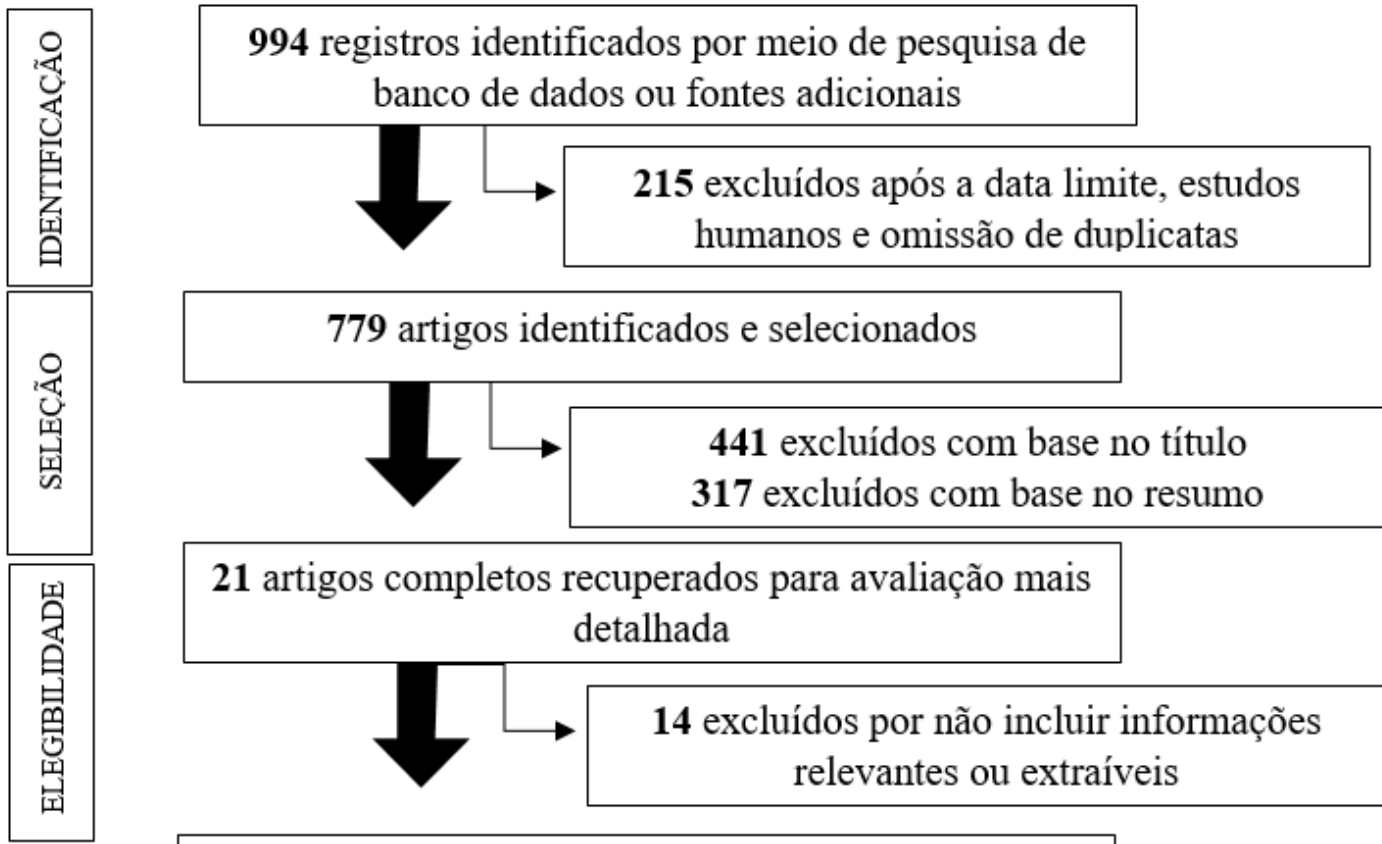

\section{1 artigos completos recuperados para avaliação mais} detalhada

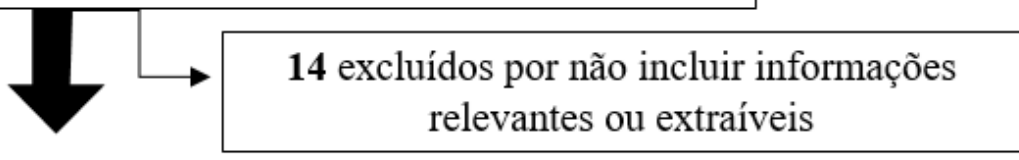

7 selecionados para revisão integrativa.

Fonte: Autores.

Visando analisar e compreender de forma integral os estudos incluídos, dados extraídos dos artigos foram descritos em duas tabelas. A Tabela 1 reporta características gerais das publicações, descrevendo o título original, autoria e ano de publicação. A Tabela 2 descreve dados relacionados ao delineamento da pesquisa, revista de publicação, objetivos do estudo e método.

Tabela 1. Características gerais dos artigos.

\begin{tabular}{|c|c|c|}
\hline Autores & Ano & Título \\
\hline 1. Hérnandez et al. & 2015 & $\begin{array}{l}\text { Tratamiento de las úlceras crónicas de las piernas con láser de Er: YAG y } \\
\text { tecnología RecoSMA }\end{array}$ \\
\hline 2. Palagi et al. & 2015 & $\begin{array}{l}\text { Impact of low-level laser therapy on the dynamics of pressure ulcer-induced } \\
\text { changes considering an infectious agent and cathelicidin LL- } 37 \text { concentration: } \\
\text { preliminary study }\end{array}$ \\
\hline 3. De Bortoli et al. & 2016 & $\begin{array}{l}\text { A Efetividade Do Laser Associado A Diferentes Tipos De Curativos Na } \\
\text { Cicatrização De Úlceras De Pressão }\end{array}$ \\
\hline 4. Machado et al. & 2017 & Low-level laser therapy in the treatment of pressure ulcers: systematic review \\
\hline 5. Bernardes et al. & 2018 & $\begin{array}{l}\text { Efeitos da laserterapia no tratamento de lesões por pressão: uma revisão } \\
\text { sistemática }\end{array}$ \\
\hline 6. Tadaraj et al. & 2018 & $\begin{array}{l}\text { Effect of laser therapy on expression of angio- and fibrogenic factors, and } \\
\text { cytokine concentrations during the healing process of human pressure ulcers }\end{array}$ \\
\hline 7. Polachini et al. & 2019 & $\begin{array}{l}\text { Efeitos da fotobioestimulação e estimulação elétrica na cicatrização e qualidade } \\
\text { de vida de indivíduos com úlceras cutâneas: terapias isoladas e associadas }\end{array}$ \\
\hline
\end{tabular}


Tabela 2. Características específicas de cada estudo.

\begin{tabular}{|c|c|c|c|c|}
\hline Artigo & Delineamento & Revista & bjetivo & Método \\
\hline 1 & Coorte prospectiva & $\begin{array}{l}\text { Cirugía Plástica } \\
\text { Ibero- } \\
\text { Latinoamericana }\end{array}$ & $\begin{array}{l}\text { Avaliar os resultados alcançados na } \\
\text { cicatrização de UP em membros } \\
\text { inferiores usando um Erbium:YAG } \\
\text { com tecnologia RecoSMA. }\end{array}$ & $\begin{array}{l}\text { Irradiação de laser Er:YAG com } \\
\text { tecnologia RecoSMA acoplada. }\end{array}$ \\
\hline 2 & Relato de Caso & $\begin{array}{l}\text { Revista da Escola de } \\
\text { Enfermagem da USP }\end{array}$ & $\begin{array}{l}\text { A finalidade do estudo foi a de } \\
\text { acompanhar o processo } \\
\text { cicatrização de } \\
\text { paciente crítico. }\end{array}$ & Relato de caso \\
\hline 3 & $\begin{array}{l}\text { Estudo Clínico } \\
\text { Randomizado }\end{array}$ & $\begin{array}{l}\text { Evidência - Ciência e } \\
\text { Biotecnologia }\end{array}$ & $\begin{array}{l}\text { Verificar a efetividade do uso da } \\
\text { laserterapia associada a diferentes } \\
\text { tipos de curativos em pacientes de } \\
\text { um hospital universitário }\end{array}$ & $\begin{array}{l}\text { Grupo 1- } \text { Laser AlGaInP (2 } \\
\text { pacientes). } \\
\text { Grupo 2- } \quad \text { Laser } \\
\begin{array}{l}\text { associado a loção oleosa } \\
\text { pacientes) }\end{array}\end{array}$ \\
\hline 4 & $\begin{array}{l}\text { Revisão } \\
\text { Sistemática }\end{array}$ & $\begin{array}{l}\text { Lasers in Medical } \\
\text { Science }\end{array}$ & $\begin{array}{l}\text { Avaliar os efeitos do LLT } \\
\text { comparados aos de outros tipos de } \\
\text { laser, outro tipo de intervenção, } \\
\text { placebo ou grupo controle no } \\
\text { tratamento de UP em humanos por } \\
\text { meio de uma revisão sistemática de } \\
\text { ensaios clínicos randomizados. }\end{array}$ & Revisão Sistemática \\
\hline 5 & $\begin{array}{l}\text { Revisão } \\
\text { Sistemática }\end{array}$ & Rev Cuid & $\begin{array}{l}\text { Estudar a eficácia da laserterapia } \\
\text { no processo de cicatrização de } \\
\text { lesões por pressão. Materiais }\end{array}$ & Revisão Sistemática \\
\hline 6 & $\begin{array}{l}\text { Estudo Clínico } \\
\text { Randomizado }\end{array}$ & $\begin{array}{l}\text { International Journal } \\
\text { of Medical Sciences }\end{array}$ & $\begin{array}{l}\text { Avaliar o efeito da irradiação laser } \\
\text { em diferentes comprimentos de } \\
\text { onda na expressão de fatores de } \\
\text { crescimento selecionados e } \\
\text { mediadores inflamatórios em } \\
\text { estágios específicos do processo de } \\
\text { cicatrização de feridas. }\end{array}$ & 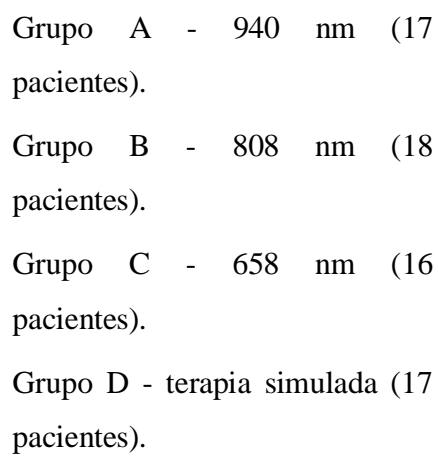 \\
\hline 7 & $\begin{array}{l}\text { Estudo Clínico } \\
\text { Randomizado }\end{array}$ & Saúde (Santa Maria) & $\begin{array}{l}\text { Investigar os efeitos de } \\
\text { intervenções fisioterapêuticas } \\
\text { isoladas e associadas com laser de } \\
\text { baixa potência e microcorrente no } \\
\text { reparo cicatricial e qualidade de } \\
\text { vida em indivíduos com úlceras } \\
\text { cutâneas de diferentes etiologias. }\end{array}$ & $\begin{array}{l}\text { Grupo 1- laser ( } 3 \text { pacientes) } \\
\text { Grupo 2- microcorrente } \\
\text { pacientes), } \\
\text { Grupo 3- laser associado a } \\
\text { microcorrente (3 pacientes). }\end{array}$ \\
\hline
\end{tabular}


O estudo de Hernández et al. (2015) realizou uma coorte prospectiva com 17 pacientes com média de idade de 69 anos, tratados com laser de Er:YAG com uso da tecnologia RecoSMA, as sessões tiveram dose de 3,2 $\mathrm{J} / \mathrm{cm}^{2}$, em pulsos de 3 Hz e 3 passagens de laser na superfície (a própria úlcera e o tecido circundante), em 1 sessão semanalmente, até um máximo de 3 meses. Neste estudo, as úlceras crônicas tratadas apresentaram cicatrização completa ou redução de sua extensão em mais de $50 \%$ de sua superfície inicial em mais de 50\% dos pacientes. Os autores relataram que o tratamento com laser é de simples aplicação e não observaram complicações durante a evolução de sua aplicação nos pacientes do grupo de estudo (Hernández et al., 2015).

O relato de caso de Palagi et al. (2015) descreve o relato de um caso de um paciente de 57 anos, foi utilizado o laser Alumínio-Gálio-Índio-Fósforo (AlGaInP), com um comprimento de onda de $660 \mathrm{~nm}$ (Po - 30Wpico), com emissão de luz contínua e pulsada uma vez ao dia, três vezes na semana, por um período de cinco semanas consecutivas, totalizando 15 aplicações. Nas bordas da lesão aplicou-se de forma pontual com $4 \mathrm{~J} / \mathrm{cm}^{2}$ e a uma distância de $1 \mathrm{~cm}$ entre os pontos. No centro da UP a FBM foi realizada com laser de baixa potência irradiado em modo varredura, com a ponteira do equipamento laser a uma distância de $1 \mathrm{~cm}$ do tecido, portanto no modo quase contato. Foi observada uma redução nas dimensões da lesão de $7 \mathrm{~cm}$ para $1,5 \mathrm{~cm}$ de comprimento e de $6 \mathrm{~cm}$ para $1,1 \mathrm{~cm}$ de largura, além do aumento do tecido epitelial e de granulação, diminuição da secreção e odor. Os autores concluíram que a intervenção com a FBM acelerou a proliferação tecidual e aumentou a vascularização local, com formação de tecido de granulação por promover rápida cicatrização da lesão, destacando essa terapêutica como uma alternativa eficaz no tratamento de UP em um paciente crítico (Palagi et al., 2015).

O estudo de De Bortoli et al. (2016) realizou um estudo clínico randomizado com dois grupos compostos por 3 úlceras cada, a média de idade dos pacientes incluídos foi de 62,2 anos. Na FBM utilizou-se o laser AlGaInP, $660 \mathrm{~nm}, 4 \mathrm{~J} / \mathrm{cm}^{2}$, uma vez ao dia por 10 dias em ambos os grupos. O laser foi associado a dois tipos de curativos, no grupo 1 o uso de curativo com solução de papaína a 10\%, enquanto no grupo 2 associado ao uso de curativo com loção oleosa à base de ácidos graxos essenciais (AGE). Ao se analisarem os resultados dos dois diferentes tipos de curativos associados ao uso de laser, observou-se que as feridas nas quais se utilizou a loção oleosa à base de AGE associados a FBM apresentaram maior redução de área e dos aspectos físicos da pele, como cor, vascularização e ausência de tecido desvitalizado nas bordas. Concluiu-se que a FBM, independentemente do tipo de curativo utilizado, apresenta resultados benéficos aos pacientes (De Bortoli et al., 2016).

O estudo de Machado et al. (2017) realizou uma revisão sistemática de literatura até maio de 2016, incluindo 4 artigos que descreveram 210 pacientes. Resultados significativos foram observados na terapia de fotobiomodulação (FBM) com LBP com comprimento de onda de $658 \mathrm{~nm}$ (vermelho) e dose de $4 \mathrm{~J} / \mathrm{cm}^{2}$ tanto no percentual de cicatrização das úlceras quanto no total de úlceras cicatrizadas ao final no período de acompanhamento. Os autores da revisão não encontraram nenhuma outra evidência com diferentes comprimento de onda além dos $658 \mathrm{~nm}$. Além disso, não foi encontrada diferença significativa quando o LBI foi usado com um cluster de sonda em UP (Machado et al., 2017).

O estudo de Bernardes et al. (2018) realizou uma revisão sistemática de literatura com corte temporal entre os anos de 2001 e 2017, incluindo 11 artigos nas línguas inglês e português. A FBM demostrou ser capaz de reduzir o número de colônias bacterianas presentes em UP, em condições experimentais. Uma vez que as bactérias não absorvem luz visível, a utilização de um fotossensibilizador específico que absorva o laser de baixa potência, gerando a produção de espécies reativas de oxigênios (EROS) por processos fotoquímicos, que promovem efetiva ação antimicrobiana na Terapia Fotodinâmica (Photodynamic Therapy). Os autores destacaram que ainda não existem definições consensuais em relação à dose e ao comprimento de onda mais indicados para o tratamento de lesões por pressão e corroboram com o estudo de Machado et al. (2017) de que a evidência atual apontam que fotobiomodulação com laser de baixa potência com $658 \mathrm{~nm}$ e dose de $4 \mathrm{~J} / \mathrm{cm}^{2}$ são os mais eficientes para o tratamento das mesmas (De Oliveira Bernardes \& Regina Jurado, 2018).

O estudo de Taradaj et al. (2018) realizou um estudo clínico randomizado com 67 pacientes com media de idade de 70 
anos em 4 grupos distintos. Pacientes de todos os grupos receberam terapia a laser uma vez por dia, 5 vezes por semana durante 1 mês. Os autores se utilizaram de lasers de baixa potência semicondutores (GaAlAs) emitiram radiação contínua em comprimentos de onda separados de $940 \mathrm{~nm}$ (grupo A), $808 \mathrm{~nm}$ (grupo B) e $658 \mathrm{~nm}$ (grupo C), irradiados a uma distância de 2 $\mathrm{cm}$ da ferida. A duração de um único protocolo dependia do tamanho da ferida, e a terapia foi ajustada para obter uma dose média de $4 \mathrm{~J} / \mathrm{cm}^{2}$. No grupo D (placebo), a terapia a laser foi aplicada da mesma maneira, mas o aparelho estava desligado durante cada sessão. O estudo concluiu que a terapia eficaz de UP está ligada à irradiação de laser em um comprimento de onda de $658 \mathrm{~nm}$ (Taradaj et al., 2018).

O estudo de Polachini et al. (2019) realizou um estudo clínico randomizado com 9 pacientes distribuídos em três grupos, no grupo 1 utilizou-se LBP, no grupo 2 microcorrente e no grupo 3 associou-se ambos; com aplicação de laser AlGaInP $660 \mathrm{~nm}$ de emissão em modo contínuo a $4 \mathrm{~J} / \mathrm{cm}^{2}$ e microcorrente ( $\mathrm{f}=130 \mathrm{~Hz} ; \mathrm{i}=300 \mu \mathrm{A}$; por 30 minutos), totalizando 15 sessões em 45 dias. Os autores observaram uma redução significativa nas áreas de todas as úlceras cutâneas estimuladas com o laser e/ou microcorrente reduziram e descreveram a cicatrização completa em duas delas. Os autores concluíram que ambas as terapêuticas associadas ou não facilitaram o processo de cicatrização e melhoraram a qualidade de vida dos pacientes. Esses achados contribuem para a prática clínica uma vez que é importante avaliar os efeitos de recursos fisioterapêuticos nãoinvasivos e indolores, quando associados ou não, afim de melhorar a terapêutica utilizada em indivíduos com úlceras cutâneas em benefício da qualidade de vida dos mesmos (Polachini et al., 2019).

\section{Conclusão}

Conclui-se que a fotobiomodulação com laser de baixa potência no tratamento de úlceras por pressão, associado ou não com terapias adjuvantes, como curativos a base de ácidos graxos e microcorrente são eficazes no tratamento das lesões. A eficácia foi descrita em todos os grupos avaliados com lasers de baixa potência com comprimento de onda na faixa de $660 \mathrm{~nm}$ (vermelho) e dose de $4 \mathrm{~J} / \mathrm{cm}^{2}$. As diversas abordagens denotam o cunho heterogêneo dos estudos, sendo que não há uma padronização efetiva do tipo de laser empregado, bem como do tempo de exposição e periodicidade dos mesmos. Mais estudos são necessários com intuito de sistematizar e quantificar as abordagens, o que culminaria numa padronização da indicação da fotobiomodulação, resultando na melhora da qualidade de vida desses pacientes pela redução das possíveis complicações, melhora da cicatrização e vascularização no local afetado, culminando na redução dos gastos devido a internação hospitalar.

\section{Referências}

Advisory, T. N. P. U. (1989). Pressure ulcers prevalence, cost and risk assessment: consensus development conference statement. Decubitus, 2(2), 24-28.

Bauer, K., Rock, K., Nazzal, M., Jones, O., \& Qu, W. (2016). Pressure Ulcers in the United States' Inpatient Population From 2008 to 2012: Results of a Retrospective Nationwide Study. Ostomy/Wound Management, 62(11), 30-38. http://www.ncbi.nlm.nih.gov/pubmed/27861135

Bhattacharya, S., \& Mishra, R. K. (2015). Pressure ulcers: Current understanding and newer modalities of treatment. Indian Journal of Plastic Surgery, 48(01), 004-016. https://doi.org/10.4103/0970-0358.155260

Botelho, L. L. R., Cunha, C. C. de A., \& Macedo, M. (2011). O Método Da Revisão Integrativa Nos Estudos Organizacionais. Gestão e Sociedade, 5(11), 121. https://doi.org/10.21171/ges.v5i11.1220

Brauncajs, M., Ksiąszczyk, K., Lewandowska-Polak, A., Gorzela, K., \& Grzegorczyk, J. (2018). Impact of low-level laser therapy on the dynamics of pressure ulcer-induced changes considering an infectious agent and cathelicidin LL-37 concentration: a preliminary study. Advances in Dermatology and Allergology, 35(6), 582-586. https://doi.org/10.5114/ada.2018.77609

Cannon, B. C., \& Cannon, J. P. (2004). Management of pressure ulcers. American Journal of Health-System Pharmacy: AJHP: Official Journal of the American Society of Health-System Pharmacists, 61(18), 1895-1905; quiz 1906-1907. http://www.ncbi.nlm.nih.gov/pubmed/15487879

Castro, M. F. de, Barbosa, L. R. P., \& Silva, L. L. da. (2020). Ação da terapia a laser de baixa intensidade na cicatrização de ulcerações diabéticas. Research, Society and Development, 9(10), e6239109109. https://doi.org/10.33448/rsd-v9i10.9109

De Bortoli, I., Prato, A. L., \& Kroth, A. (2016). A Efetividade Do Laser Associado A Diferentes Tipos De Curativos Na Cicatrização De Úlceras De Pressão. Evidência - Ciência e Biotecnologia, 16(1), 45-59. https://doi.org/10.18593/eba.v16i1.9774 
De Oliveira Bernardes, L., \& Regina Jurado, S. (2018). Efeitos da laserterapia no tratamento de lesões por pressão: uma revisão sistemática. Revista Cuidarte, 9(3), 1-12. https://doi.org/10.15649/cuidarte.v9i3.574

De Souza, Í. P., Androlage, J. S., Bellato, R., \& Barsaglini, R. A. (2019). A qualitative approach to rare genetic diseases: An integrative review of the national and international literature. Ciencia e Saude Coletiva, 24(10), 3683-3700. https://doi.org/10.1590/1413-812320182410.17822019

García-Fernández, F. P., Agreda, J. J. S., Verdú, J., \& Pancorbo-Hidalgo, P. L. (2014). A New Theoretical Model for the Development of Pressure Ulcers and Other Dependence-Related Lesions. Journal of Nursing Scholarship, 46(1), 28-38. https://doi.org/10.1111/jnu.12051

Health, W. K. (2019). Photobiomodulation Therapy for Wound Care: A Potent, Noninvasive, Photoceutical Approach. Adv Skin Wound Care, 32 , ASWC0419.

Hernández, E., Khomchenko, V., Sola, A., Pikirenia, I. I., Alcolea, J. M., \& Trelles, M. A. (2015). Tratamiento de las úlceras crónicas de las piernas con láser de Er: YAG y tecnología RecoSMA. Cirugía Plástica Ibero-Latinoamericana, 41(3), 271-282. https://doi.org/10.4321/S0376-78922015000300007

Higgins, J. P. T., \& Green, S. (2011). Cochrane Handbook for Systematic Reviews of Interventions. The Cochrane Collaboration. www.handbook.cochrane.org

Li, S., Wang, C., Wang, B., Liu, L., Tang, L., Liu, D., Yang, G., \& Zhang, L. (2018). Efficacy of low-level light therapy for treatment of diabetic foot ulcer: A systematic review and meta-analysis of randomized controlled trials. Diabetes Research and Clinical Practice, 143, 215-224. https://doi.org/10.1016/j.diabres.2018.07.014

Lima, A. C. B., \& Guerra, D. M. (2011). Avaliação do custo do tratamento de úlceras por pressão em pacientes hospitalizados usando curativos industrializados. Ciência \& Saúde Coletiva, 16(1), 267-277. https://doi.org/10.1590/S1413-81232011000100029

Lucas, C., Van Gemert, M. J. C., \& De Haan, R. J. (2003). Efficacy of low-level laser therapy in the management of stage III decubitus ulcers: A prospective, observer-blinded multicentre randomised clinical trial. Lasers in Medical Science, 18(2), 72-77. https://doi.org/10.1007/s10103-003-0259-5

Machado, R. S., Viana, S., \& Sbruzzi, G. (2017). Low-level laser therapy in the treatment of pressure ulcers: systematic review. Lasers in Medical Science, 32(4), 937-944. https://doi.org/10.1007/s10103-017-2150-9

Mervis, J. S., \& Phillips, T. J. (2019). Pressure ulcers: Prevention and management. Journal of the American Academy of Dermatology, 81(4), 893-902. https://doi.org/10.1016/j.jaad.2018.12.068

Monteiro, D. da R., Báo, A. C. P., Souza, T. de C., \& Tanaka, R. Y. (2020). Efeito da terapia a laser no tratamento de lesões por pressão em pacientes adultos: revisão integrativa. Research, Society and Development, $9(10)$, e6589109094. https://doi.org/10.33448/rsd-v9i10.9094

Mosca, R. C., Ong, A. A., Albasha, O., Bass, K., \& Arany, P. (2019). Photobiomodulation Therapy for Wound Care. Advances in Skin \& Wound Care, 32(4), 157-167. https://doi.org/10.1097/01.ASW.0000553600.97572.d2

Mustoe, T. A., O'Shaughnessy, K., \& Kloeters, O. (2006). Chronic Wound Pathogenesis and Current Treatment Strategies: A Unifying Hypothesis. Plastic and Reconstructive Surgery, 117(SUPPLEMENT), 35S-41S. https://doi.org/10.1097/01.prs.0000225431.63010.1b

National Pressure Ulcer Advisory and Pan Pacific Pressure Injury Alliance. (2015). Prevention and Treatment of Pressure Ulcers : Quick Reference Guide. In Clinical Practice Guideline. https://www.epuap.org/wp-content/uploads/2016/10/quick-reference-guide-digital-npuap-epuap-pppia-jan2016.pdf

Palagi, S., Severo, I. M., Menegon, D. B., \& Lucena, A. de F. (2015). Laser therapy in pressure ulcers: Evaluation by the Pressure Ulcer Scale for Healing and Nursing Outcomes Classification. Revista Da Escola de Enfermagem, 49(5), 826-833. https://doi.org/10.1590/S0080-623420150000500017

Pereira, A. S., Shitsuka, D. M., Parreira, F. J., \& Shitsuka, R. (2018). Método Qualitativo, Quantitativo ou Quali-Quanti. In Metodologia da Pesquisa Científica. https://repositorio.ufsm.br/bitstream/handle/1/15824/Lic_Computacao_Metodologia-Pesquisa-Cientifica.pdf?sequence=1.

Polachini, C. R. N., Berni, V. B., Bellé, J. M., Oliveira, D. F. de, \& Saccol, M. F. (2019). Efeitos da fotobioestimulação e estimulação elétrica na cicatrização e qualidade de vida de indivíduos com úlceras cutâneas: terapias isoladas e associadas. Saúde (Santa Maria), 45(3), 1-18. https://doi.org/10.5902/2236583439675

Qaseem, A., Humphrey, L. L., Forciea, M. A., Starkey, M., \& Denberg, T. D. (2015). Treatment of pressure ulcers: A clinical practice guideline from the American College of Physicians. Annals of Internal Medicine, 162(5), 370-379. https://doi.org/10.7326/M14-1568

Sardo, P. M. G., Simões, C. S. O., Alvarelhão, J. J. M., Simões, J. F. F. L., Machado, P. A. P., Amado, F. M. L., Amaro, A. J. M., \& Melo, E. M. O. P. de. (2016). Analyses of pressure ulcer incidence in inpatient setting in a Portuguese hospital. Journal of Tissue Viability, 25(4), 209-215. https://doi.org/10.1016/j.jtv.2016.08.003

Strazzieri-Pulido, K. C., S. González, C. V., Nogueira, P. C., Padilha, K. G., \& G. Santos, V. L. C. (2019). Pressure injuries in critical patients: Incidence, patient-associated factors, and nursing workload. Journal of Nursing Management, 27(2), 301-310. https://doi.org/10.1111/jonm.12671

Taly, A. B., Sivaraman Nair, K. P., Murali, T., \& John, A. (2004). Efficacy of multiwavelength light therapy in the treatment of pressure ulcers in subjects with disorders of the spinal cord: A randomized double-blind controlled trial. Archives of Physical Medicine and Rehabilitation, 85(10), 1657-1661. https://doi.org/10.1016/j.apmr.2004.03.028

Taradaj, J., Shay, B., Dymarek, R., Sopel, M., Walewicz, K., Beeckman, D., Schoonhoven, L., Gefen, A., \& Rosińczuk, J. (2018). Effect of laser therapy on expression of angio- and fibrogenic factors, and cytokine concentrations during the healing process of human pressure ulcers. International Journal of Medical Sciences, 15(11), 1105-1112. https://doi.org/10.7150/ijms.25651

Thomé Lima, A. M. C., da Silva Sergio, L. P., da Silva Neto Trajano, L. A., de Souza, B. P., da Motta Mendes, J. P., Cardoso, A. F. R., Figueira, C. P., dos Anjos Tavares, B., Figueira, D. S., Mencalha, A. L., Trajano, E. T. L., \& de Souza da Fonseca, A. (2019). Photobiomodulation by dual-wavelength low-power laser effects on infected pressure ulcers. Lasers in Medical Science, 35(3), 651-660. https://doi.org/10.1007/s10103-019-02862-w 
Research, Society and Development, v. 10, n. 2, e32810212597, 2021

(CC BY 4.0) | ISSN 2525-3409 | DOI: http://dx.doi.org/10.33448/rsd-v10i2.12597

Wada, A., Teixeira Neto, N., \& Ferreira, M. C. (2010). Úlceras por pressão. Revista de Medicina, 89(3/4), 170. https://doi.org/10.11606/issn.16799836.v89i3/4p170-177

Zhao, R., Liang, H., Clarke, E., Jackson, C., \& Xue, M. (2016). Inflammation in Chronic Wounds. International Journal of Molecular Sciences, 17(12), 2085. https://doi.org/10.3390/ijms 17122085 